\title{
The HERMES Recoil Detector
}

\author{
R. Kaiser (for the HERMES Collaboration) \\ Dept. of Physics and Astronomy, University of Glasgow
}

\begin{abstract}
The HERMES Collaboration is installing a new Recoil Detector to upgrade the spectrometer for measurements of hard exclusive electron/positron scattering reactions, in particular deeply virtual Compton scattering. These measurements will provide access to generalised parton distributions and hence to the localisation of quarks inside hadrons and to their orbital angular momentum. The HERMES Recoil Detector consists of three active components: a silicon detector surrounding the target cell inside the beam vacuum, a scintillating fibre tracker and a photon detector consisting of three layers of tungsten/scintillator. All three detectors are located inside a solenoidal magnetic field of 1 Tesla. The Recoil Detector was extensively tested with cosmic muons over the summer of 2005 and is being installed in the winter of 2005/6 for data taking until summer 2007.
\end{abstract}

Keywords: deeply virtual Compton scattering, recoil proton, generalised parton distributions

PACS: 14.20.Dh, 13.85.Hd, 29.40.Mc, 29.40.Wk, 29.30.Aj

\section{INTRODUCTION}

Generalised parton distributions (GPDs) offer a way to unify the previously disjunct pictures of the nucleon given by parton distribution functions on the one hand and nucleon form factors on the other. For the first time GPDs may provide detailed information on the localisation of partons inside hadrons and access to their orbital angular momentum [1]. GPDs can be accessed experimentally through the measurement of hard exclusive reactions, the cleanest of which is deeply virtual Compton scattering (DVCS) [2].

In the reaction $e p \rightarrow e^{\prime} \gamma p$ at the beam energy of HERMES $(27.5 \mathrm{GeV})$, bremsstrahlung (Bethe-Heitler) dominates over DVCS in most of the kinematic region. However, measurable asymmetries in beam spin and beam charge arise from the interference of both processes. The beam spin asymmetry is proportional to the imaginary part of the DVCS amplitude, while the beam charge asymmetry is proportional to the real part of the DVCS amplitude. Both can in turn be expressed in terms of GPDs.

\section{THE RECOIL DETECTOR}

The HERMES Recoil Detector has been designed to upgrade the HERMES spectrometer for the measurement of hard exclusive reactions, in particular DVCS. Its objectives are the detection and identification of recoil protons and the rejection of background events.

The Recoil Detector consists of three active components: a silicon strip detector surrounding the target cell inside the beam vacuum, a scintillating fibre tracker and a photon detector consisting of three layers of tungsten/scintillator. All three detectors are are located inside a solenoidal magnetic field of 1 Tesla. Together these detectors cover most of the kinematic region for DVCS/Bethe-Heitler events. The detector and its 
anticipated performance are described in detail in the Technical Design Report [3].

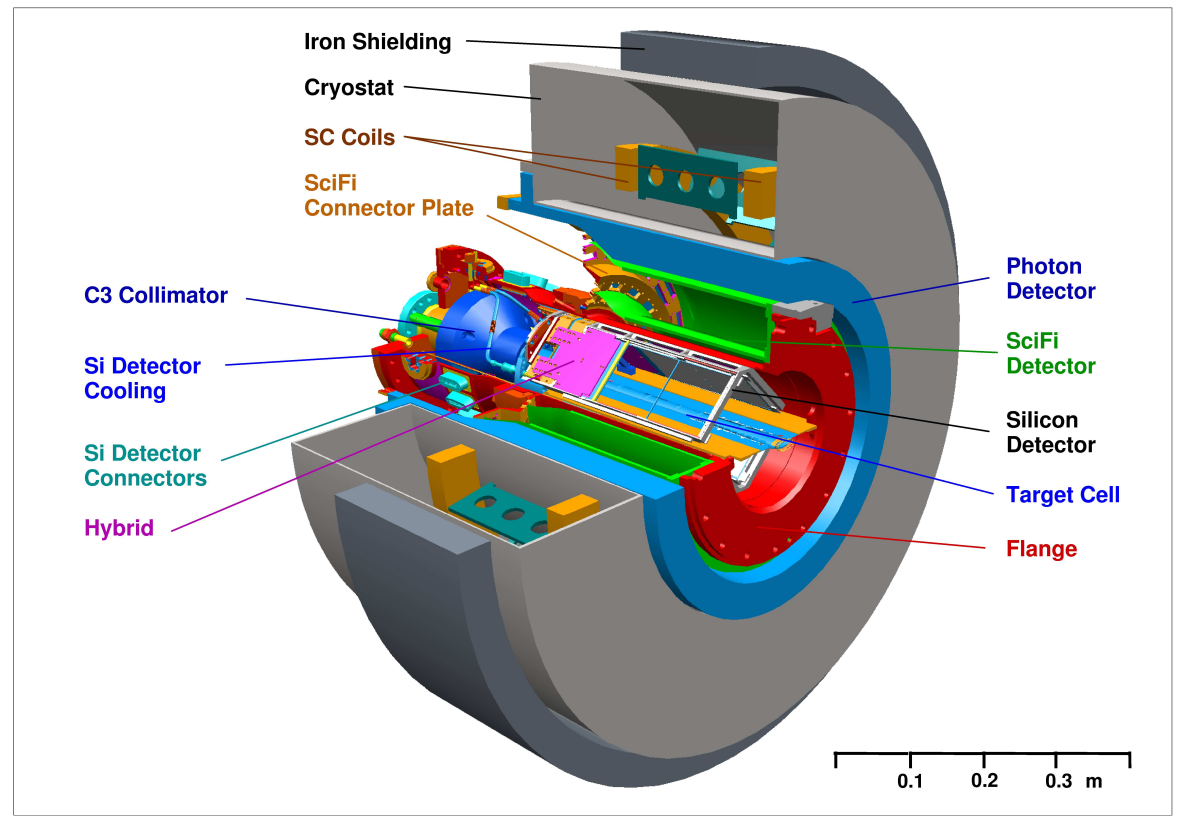

FIGURE 1. Schematic overview of the HERMES Recoil Detector.

\section{Silicon Strip Detector}

The silicon detector consists of 16 double sided sensors based on the TTT design by Micron Semiconductors [4], with 128 strips per side and a strip width of $758 \mu \mathrm{m}$. The detector is located inside the HERA beam vacuum, only centimeters from the 27.5 . GeV electron/positron beam. It measures the momentum of recoil protons in the range 135 $400 \mathrm{MeV} / \mathrm{c}$ via their energy deposition and also provides space points for the tracking of minimum ionising particles (MIPs). The required dynamic range of 1-70 MIPs was realised by a charge division readout using two HELIX 3.0 channels per silicon strip. The sensors have been calibrated with low energy protons at the Erlangen Tandem accelerator to better than 2\%. The signal/noise ratio for MIPs has been measured at an electron test beam at DESY to be 6.5, corresponding to a MIP detection efficiency of close to $99 \%$.

\section{Scintillating Fibre Tracker}

Proton momenta above $250 \mathrm{MeV} / \mathrm{c}$ are measured through the deflection of their tracks in the solenoidal 1T magnetic field. To this end two additional space points are provided by a scintillating fibre tracker (SFT). The SFT consists of two barrels, each of which has 2 parallel and 2 stereo layers $\left(10^{\circ}\right)$ of $1 \mathrm{~mm}$ Kuraray SCSF-78 fibres. The barrels are constructed as self-supporting structures to minimise the amount of material traversed by the particles. The detector is read out via $4 \mathrm{~m}$ long light guides and 64 channel 
Hamamatsu PMTs. The readout electronics is based on the GASSIPLEX chip, with additional fast timing information provided by a TDC readout coupled to the signals from dynode 12 of the PMTs. The positioning of the fibres within the barrels was mapped at the DESY electron testbeam with a precision of better than $80 \mu \mathrm{m}$ for the entire SFT. In addition to the tracking information the energy deposition in the fibres also provides pion/proton particle identification up to about $450 \mathrm{MeV} / \mathrm{c}$.

\section{Photon Detector}

The photon detector is a 3-layer tungsten/scintillator calorimeter with $2 \mathrm{~cm}$-wide scintillator strips that are read out using wavelength shifting fibres. The innermost strips are parallel to the beam, the two further layers have stereo angles of $\pm 45^{\circ}$. The detection of photons, especially those originating from $\Delta^{+}$-decays, will allow the further reduction of non-exclusive background events. Additionally, the photon detector will provide a cosmic ray trigger for test and alignment measurements and serve for additional pion/proton particle identification at higher momenta.

\section{COSMICS TEST EXPERIMENT}

Between April and September 2005 the completely assembled Recoil Detector was tested using cosmic muons. This test provided important insights in some hardware issues and allowed the integration of the entire detector into the HERMES data acquisition system. A total of 2 TByte of raw data was collected that are being used for the calibration of SFT and photon detector as well as for the development of software for the internal alignment of the detector with straight tracks.

After a rough alignment, the residuals from cosmic tracks were measured to be 0.27 $\mathrm{mm}$ for the silicon detector and $0.35 \mathrm{~mm}$ for the SFT, about $20 \%$ higher than what would be expected for a perfectly aligned detector. Efficiencies for SFT and photon detector were in agreement with test beam measurements, but the MIP detection efficiency for the silicon strip detector was with $80-90 \%$ significantly lower. This is likely due to the high common mode noise present in the test experiment. The electrical set-up of the detector was revised and the noise level was reduced to the level known from previous test beam measurements. Efficiencies of the same order as in the test beam measurements are therefore expected for the final setup.

The detector will be installed in the HERMES experiment in the winter of 2005/6, for data taking from February 2006 to June 2007.

\section{REFERENCES}

1. X. Ji, Phys.Rev.Lett. 78 (1997) 610; Phys.Rev. D 55 (1997) 7114

2. V. A. Korotkov, W.-D. Nowak, Nucl.Phys. A711 (2002) 175

3. HERMES Collab. (R.Kaiser ed.), The HERMES Recoil Detector Technical Design Report, DESY PRC 02-01, HERMES Internal Note 02-033, 2002

4. http://www.micronsemiconductor.co.uk/pdf/ttt.pdf 